\title{
On the implementation of a recently proposed dosimetric formalism to a robotic radiosurgery system
}

\author{
E. Pantelis ${ }^{\text {a) }}$ \\ Medical Physics Laboratory, Medical School, University of Athens, 75 Mikras Asias, 11527 Athens, Greece \\ and CyberKnife Center, Iatropolis-Magnitiki Tomografia, 54-56 Ethnikis-Antistaseos, 152 31, \\ Athens, Greece \\ A. Moutsatsos and K. Zourari \\ Medical Physics Laboratory, Medical School, University of Athens, 75 Mikras Asias, 11527 Athens, Greece \\ W. Kilby \\ Accuray Inc., 13010 Chesapeake Terrace, Sunnyvale, California 94089 \\ C. Antypas \\ First Department of Radiology, Aretaieion Hospital, Medical School, University of Athens, Athens, Greece \\ and CyberKnife Center, Iatropolis-Magnitiki Tomografia, 54-56 Ethnikis-Antistaseos, 152 31, \\ Athens, Greece \\ P. Papagiannis, P. Karaiskos, and E. Georgiou \\ Medical Physics Laboratory, Medical School, University of Athens, 75 Mikras Asias, 11527 Athens, Greece \\ L. Sakelliou \\ Department of Physics, Nuclear and Particle Physics Section, University of Athens, Panepistimioupolis, \\ Ilisia, 15771 Athens, Greece
}

(Received 11 January 2010; revised 10 March 2010; accepted for publication 31 March 2010; published 29 April 2010)

Purpose: The aim of this work is to implement a recently proposed dosimetric formalism for nonstandard fields to the calibration and small field output factor measurement of a robotic stereotactic radiosurgery system.

Methods: Reference dosimetry measurements were performed in the nonstandard, $60 \mathrm{~mm}$ diameter machine specific reference (msr) field using a Farmer ion chamber, five other cylindrical chambers with cavity lengths ranging from 16.25 down to $2.7 \mathrm{~mm}$, and alanine dosimeters. Output factor measurements were performed for the 5, 7.5, 10, and $15 \mathrm{~mm}$ field sizes using microchambers, diode detectors, alanine dosimeters, TLD microcubes, and EBT Gafchromic films. Measurement correction factors as described in the proposed formalism were calculated for the ion chamber and diode detector output factor measurements based on published Monte Carlo data. Corresponding volume averaging correction factors were calculated for the alanine output factor measurements using 3D dose distributions, measured with polymer gel dosimeters.

Results: Farmer chamber and alanine reference dosimetry results were found in close agreement, yielding a correction factor of $k_{Q_{\text {msr }}}^{f_{\text {msr }}, f_{\text {ref }}}=0.999 \pm 0.016$ for the chamber readings. These results were also found to be in agreement within experimental uncertainties with corresponding results obtained using the shorter cavity length ionization chambers. The mean measured dose values of the latter, however, were found to be consistently greater than that of the Farmer chamber. This finding, combined with an observed inverse relationship between the mean measured dose and chamber cavity length that follows the trend predicted by theoretical volume averaging calculations in the msr field, implies that the Farmer $k_{Q_{\text {msr }}}^{f_{\text {mst }}, f_{\text {ref }}}$ correction is greater than unity. Regarding the output factor results, deviations as large as 33\% were observed between the different dosimeters used. These deviations were substantially decreased when appropriate correction factors were applied to the measured microchamber, diode, and alanine values. After correction, all diode and microchamber measured output factors agreed within $1.6 \%$ with the corresponding alanine measurements, and within $3.1 \%$ with the TLD measurements. The weighted mean output factors were $0.681 \pm 0.001$, $0.824 \pm 0.001,0.875 \pm 0.001$, and $0.954 \pm 0.001$ for the $5,7.5,10$, and $15 \mathrm{~mm}$ beams, respectively. Conclusions: The comparison of Farmer chamber measurements versus alanine reference dosimetry validates the use of the former for dosimetry in the msr field of this treatment delivery system. The corresponding results of this work obtained using chambers with different cavity lengths, combined with previous literature findings, suggest that a $k_{Q \text { msr }}^{f_{\text {msr }}} f_{\text {ref }}$ Farmer chamber dose response correction factor of 1.01 may improve calibration measurement accuracy when using the proposed dosimetric formalism. The $k_{Q_{\text {msrr }}}^{f_{\text {sr }}, f_{\text {ref }}}$ correction factor is within $0.5 \%$ from unity for ion chambers with cavity lengths less than $10 \mathrm{~mm}$. Substantial improvements in small field output factor measurement accuracy can be obtained when using microchambers and diodes by applying appropri- 
ately calculated correction factors to the detector measurements according to the proposed dosimetric formalism, and their routine use is therefore recommended. (c) 2010 American Association of Physicists in Medicine. [DOI: 10.1118/1.3404289]

Key words: small fields, calibration dosimetry, output factor, alanine, CyberKnife

\section{INTRODUCTION}

Small photon fields defined by circular collimators or multileaf collimators are used routinely for stereotactic radiosurgery/radiotherapy and intensity modulated radiation therapy. Accurate dosimetry of small fields is complicated by the presence of nonequilibrium conditions, steep dose gradients, and field perturbation effects. ${ }^{1-8}$ In addition, due to their mechanical design, some treatment devices such as the CyberKnife ${ }^{\circledR}$ Robotic Radiosurgery System (Accuray Inc., Sunnyvale, CA), the Tomotherapy ${ }^{\circledR} \mathrm{Hi}-\mathrm{Art}^{\circledR}$ (Tomotherapy Inc., Madison, WI), and the Leksell GammaKnife ${ }^{\circledR}$ (Elekta Instrument $\mathrm{AB}$, Stockholm, Sweden) cannot establish the classical $100 \times 100 \mathrm{~mm}^{2}$ reference field required for calibration purposes. ${ }^{8-12}$ Therefore, these units are typically calibrated using a different field, assuming that the calibration of the detector used for dose measurement in the nonstandard field is identical to that in the classical broad-beam conditions.

Recently, a new formalism for the dosimetry of small and nonstandard treatment fields has been proposed by the IAEA for wider discussion. ${ }^{13}$ The specific formalism is presented as an extension of the formalism used in the IAEA TRS-398 code of practice ${ }^{14}(\mathrm{CoP})$ and introduces two alternative calibration routes; (a) small static field dosimetry and (b) composed field dosimetry. In the small static field route, a machine specific reference $(\mathrm{msr})$ field is defined and a correction factor is introduced that relates the response of a dosimeter in the msr field to its response under classical broad-beam conditions. An additional correction factor is used to account for response changes in small field output factor measurements with respect to the msr field. These new correction factors can be determined by Monte Carlo (MC) simulations or by intercomparison against another dosimeter for which the correction factors are known.

In this work, the small static field dosimetry route described in the new formalism ${ }^{13}$ was applied to calibration and output factor measurements using a CyberKnife System. For this treatment delivery system the, msr calibration measurement is, most commonly, performed using a Farmer type ionization chamber. Previous studies employing MC simulation suggest that the additional msr correction factor that must be applied in this situation is unity. ${ }^{13}$ However, a recent theoretical assessment ${ }^{10}$ showed that the dose gradient caused by the absence of a flattening filter across the cavity volume is on the order of $1.5 \%$ for the Farmer chamber cavity dimensions, and therefore a Farmer chamber should be considered too large for this measurement (see Fig. 1). Regarding small field output factor measurements of this system, a machine specific MC based methodology has been recently proposed to calculate correction factors for a variety of detectors which are equivalent to the correction factors proposed in the new formalism. ${ }^{3,4,15}$

The purpose of this work is to implement the small static field method of the proposed dosimetric formalism ${ }^{13}$ for calibration and small field output factor measurements of the CyberKnife System. The correction factor required in this formalism for reference dosimetry measurements is evaluated for a Farmer type ionization chamber by comparing its dose response with the corresponding response of alanine dosimeters and ionization chambers to shorter cavity lengths. Small field output factor measurements were performed using two microchambers, three different diode detectors, alanine dosimeters, TLD microcubes, and Gafchromic EBT films. Correction factors equivalent to those suggested by the dosimetric formalism for small field measurements were calculated using the aforementioned MC based methodology $y^{3,4,15}$ for the microchambers and diode detectors. The accuracy of the calculated correction factors was established by comparing the corrected output factors to the corresponding factors measured using alanine, TLD, and EBT film water equivalent dosimeters.

\section{MATERIALS AND METHODS}

\section{II.A. Dosimetric formalism}

Following the proposed dosimetric formalism, ${ }^{13}$ the absorbed dose to water $D_{w, Q_{\text {msr }}}^{f_{\text {msr }}}$ at the reference depth in the machine specific reference field $f_{\mathrm{msr}}$, with beam quality $Q_{\mathrm{msr}}$, is given by

$$
D_{w, Q_{\mathrm{msr}}}^{f_{\mathrm{msr}}}=M_{Q_{\mathrm{msr}}}^{f_{\mathrm{msr}}} \cdot N_{D, w, Q_{o}} \cdot k_{Q, Q_{o}} \cdot k_{Q_{\mathrm{msr}}}^{f_{\mathrm{msr}}, f_{\mathrm{ref}}},
$$

where $M_{Q_{\text {msr }}}^{f_{\text {ms }}}$ is the reading of the dosimeter corrected for influence quantities and $N_{D, w, Q_{o}}$ is the calibration coefficient in terms of absorbed dose to water for an ionization chamber at a reference beam quality $Q_{o}$ with standard reference field and geometry. $k_{Q, Q o}$ is the beam quality correction factor, which corrects for the differences between the reference beam quality $Q_{o}$ at the standard laboratory and the beam quality $Q$ of the reference field, $f_{\text {ref }} \cdot k_{Q_{\mathrm{msr}}}^{f_{\mathrm{mrr}} f_{\text {ref }}}$ is a chamberspecific correction factor which accounts for differences in beam geometry, beam quality, and phantom material between $f_{\text {ref }}$ and the $f_{\text {msr }}$.

The absorbed dose to water at a reference point in a clinical field $f_{\text {clin }}$ is then obtained from:

$$
D_{w, Q_{\text {clin }}}^{f_{\text {clin }}}=D_{w, Q_{\text {msr }}}^{f_{\text {msr }}} \cdot \Omega_{Q_{\text {clin }}, Q_{\mathrm{msr}}}^{f_{\text {cin }}, f_{\text {msr }}},
$$

where $Q_{\text {clin }}$ is the beam quality of $f_{\text {clin }}$ and $\Omega_{Q_{\text {clin }}, Q_{\text {msr }}}^{f_{\text {cling }}, f_{\text {msr }}}$ is the output factor that converts the absorbed dose to water at the reference point from the $f_{\text {msr }}$ to $f_{\text {clin }}$. The output factor can be 


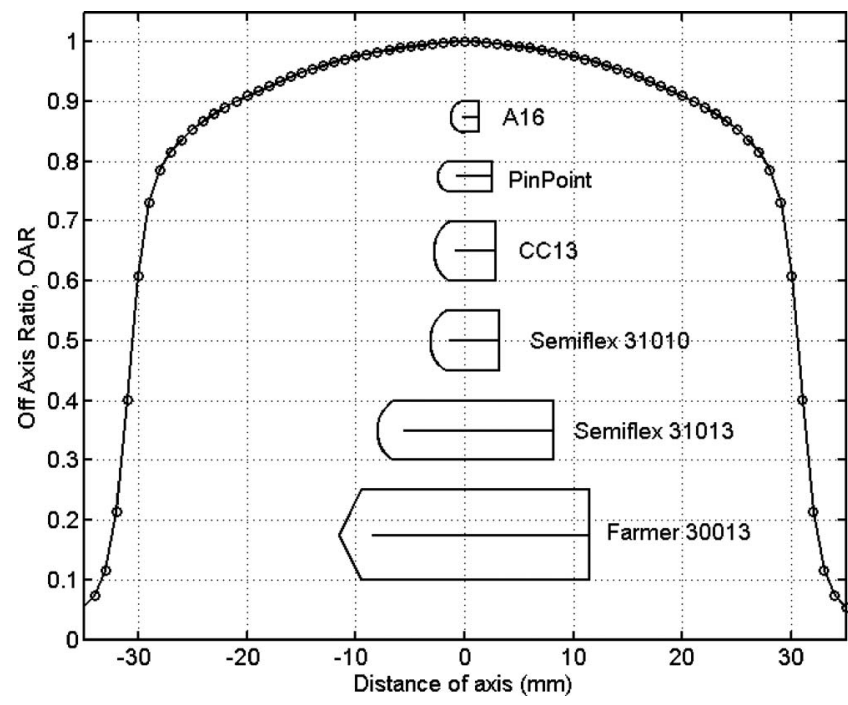

FIG. 1. The off-axis profile of the $60 \mathrm{~mm}$ fixed collimator beam measured at $50 \mathrm{~mm}$ depth in water plotted versus off-axis distance. The geometry of the air cavity of the different ion chambers used for calibration dosimetry measurements is also depicted. The dose gradient over a Farmer chamber cavity resulting from the absence of a beam flattening filter is clearly apparent.

measured as the ratio of detector readings multiplied by a correction factor $k_{Q_{\text {clin }}, f_{\text {msr }},}$, which accounts for the difference between the detector response in the $f_{\text {clin }}$ and $f_{\mathrm{msr}}$ according to

$$
\Omega_{Q_{\text {clin }}, Q_{\mathrm{msr}}}^{f_{\mathrm{min}}, f_{\mathrm{msr}}}=\frac{M_{Q_{\mathrm{clin}}}^{f_{\mathrm{clin}}}}{M_{Q_{\mathrm{msr}}}^{f_{\mathrm{msr}}}} \cdot k_{Q_{\mathrm{clin}}, Q_{\mathrm{msr}}}^{f_{\mathrm{clin}}, f_{\mathrm{msr}}} .
$$

The correction factor $k_{\mathcal{C}_{\text {clin }}, Q_{\text {msr }}}^{f_{\text {lis }}, f_{\text {sr }}}$ depends on $f_{\text {clin }}$ and the dosimetric characteristics of each detector (i.e., energy dependence, water equivalence, sensitive volume size, etc). If, for a given detector, $k_{\mathcal{Q}_{\text {clin }}, Q_{\text {msr }}}^{f_{\text {ms }}}$ is close to unity, then the ratio of the detector readings alone is a sufficiently accurate approximation to the output factor.

Correction factors with an equivalent meaning to $k_{Q_{\text {clin }}}^{f_{\text {clingr }}, \rho_{\text {msr }}}$ have been suggested in the literature using analytical equations and MC simulations, used independently or in combination with experimental measurements. ${ }^{2-4,7,16,17}$ For the CyberKnife System specifically, Francescon et al. ${ }^{3,4,15}$ performed MC simulations and measurements to quantify the dependence of these correction factors on beam energy and focal spot size for a variety of detectors and the 5, 7.5, and $10 \mathrm{~mm}$ diameter fixed collimator fields. They parameterized the observed beam energy and spot size dependence and suggested a machine specific methodology that should be followed to obtain the equivalent to $k_{Q_{\text {clin }}, Q_{\text {msr }}}^{f_{\text {lins }}, g_{\text {msr }}}$ correction factors for a series of solid state and microchamber detectors.

\section{II.B. Reference dosimetry in the msr field}

\section{II.B.1. Reference field beam quality}

The CyberKnife System uses a 6 MV photon beam with circular collimation and no flattening filter. The msr field for the CyberKnife System is defined as the $60 \mathrm{~mm}$ diameter field produced by a fixed collimator, at $800 \mathrm{~mm}$ source-

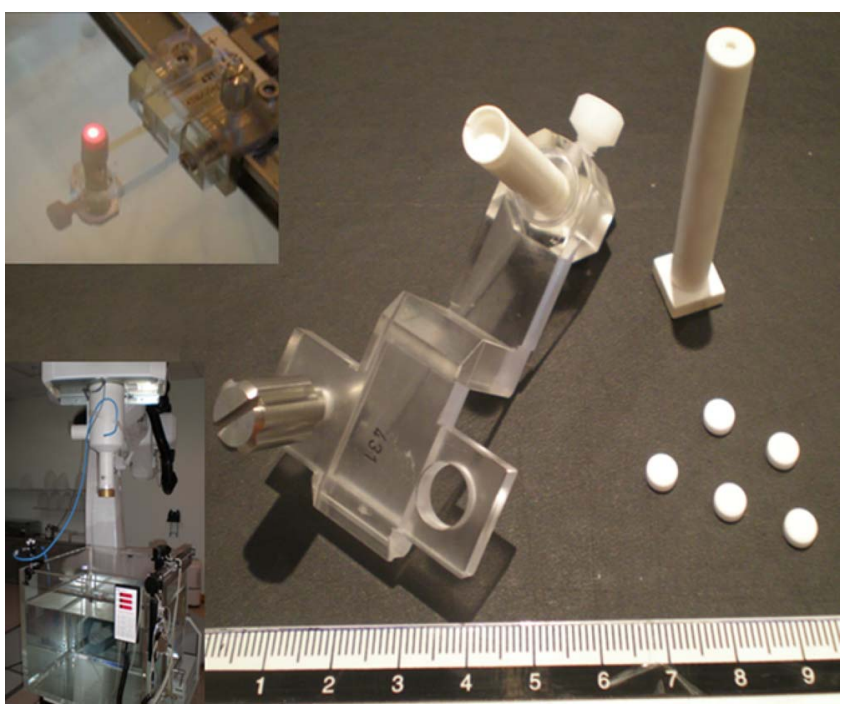

FIG. 2. Photograph of the alanine pellets, constructed holders, and experimental setup for irradiation in the CyberKnife System. The depicted ruler scale is in centimeters.

detector-distance (SDD). ${ }^{18} \mathrm{TPR}_{20 / 10}\left(f_{\mathrm{msr}}\right)$ was measured for the $f_{\mathrm{msr}}$ using a SDD of $800 \mathrm{~mm}$, which corresponds to the standard intracranial treatment distance, and of $1000 \mathrm{~mm}$ following the recommendations of TRS-398 CoP. ${ }^{14}$ An equivalent $\mathrm{TPR}_{20 / 10}$ corresponding to a hypothetical 100 $\times 100 \mathrm{~mm}^{2}$ reference field at $1000 \mathrm{~mm}$ SDD was calculated from these measurements by: (a) Substituting the value measured at $800 \mathrm{~mm} \mathrm{SDD}$ and an equivalent square field size of $54 \mathrm{~mm}\left(s_{\mathrm{eq}}=0.9 \times f_{\mathrm{msr}}\right)$ into the function proposed by Sauer ${ }^{19}$ to convert to $100 \mathrm{~mm}$ equivalent square, and (b) combining the value measured at $1000 \mathrm{~mm}$ SDD and an equivalent square field size of $67 \mathrm{~mm}\left(s_{\mathrm{eq}}=0.9 \times f_{\mathrm{msr}} \times 1000 / 800\right)$ with the TPR data tables given in BJR supplement 25 (Ref. 20) to estimate a value at $100 \mathrm{~mm}$ equivalent square. The resulting values were used to select $k_{Q, Q o}$ values for the ion chambers employed for reference dosimetry measurements in this work.

\section{II.B.2. Alanine measurements}

A batch of 40 cylindrical alanine pellets was provided by the therapy-level alanine-dosimetry service ${ }^{21}$ of the National Physical Laboratory (NPL) for this work. An additional 20 control pellets from the same batch (ten preirradiated to 10 Gy and ten nonirradiated) were also delivered. Each pellet had an outer diameter of $5 \mathrm{~mm}$ and a height of $2.5 \mathrm{~mm}$. All alanine packages were stored at constant temperature and opened just before their irradiation.

In order to minimize positioning uncertainties, a cylindrical holder was constructed from RW3 solid water material (mass density $1.045 \mathrm{~g} \mathrm{~cm}^{-3}$, electron density relative to water 1.012) and used with the MP3 motorized water phantom (PTW, Freiburg, Germany) (see Fig. 2). The outer diameter of the holder was $7 \mathrm{~mm}$ in order to fit in the No. 431 PTWTRUFIX positioning device, which is used for irradiating the 
PTW 60008 diode detector (see Fig. 2). At the tip of the holder, a cylindrical cavity of $5 \mathrm{~mm}$ diameter and $2.5 \mathrm{~mm}$ height was drilled to hold one alanine pellet. The height of the holder was $45.5 \mathrm{~mm}$ so that the tip of the holder lay at the same point as the front surface of the 60008 diode detector when placed in the water phantom using the TRUFIX positioning device.

The center of the holder was carefully aligned with the radiation central axis by first scanning with a 60008 diode placed in the TRUFIX device to find the beam center. When fine alignment was achieved, the diode was removed and replaced with the holder loaded with an alanine pellet. Since the alanine dosimeters are not waterproof a watertight sleeve of $0.1 \mathrm{~mm}$ thickness was also used. The geometrical center of the pellet was placed at $50 \mathrm{~mm}$ reference depth in water and irradiated using the $60 \mathrm{~mm}$ diameter fixed collimator and an SDD of $800 \mathrm{~mm}$. A total of 1106 monitor units (MUs) were delivered to each alanine pellet, corresponding to a nominal dose of $10 \mathrm{~Gy}$ at that depth. Five alanine pellets were irradiated serially for statistical reasons.

The readout of the alanine dosimeters was performed by the NPL primary standard dosimetry laboratory. The intensity of the $X$-band electron paramagnetic resonance spectrum was measured and converted to absorbed dose to water using the calibration of the alanine batch used in this work. The alanine dosimeters were calibrated in terms of absorbed dose to water by the NPL using a ${ }^{60} \mathrm{Co}$ gamma ray field. A beam quality correction factor of 1.006 was also applied to correct for the lower dose response of the dosimeters in megavoltage $\mathrm{x}$-ray beams compared to that in ${ }^{60} \mathrm{Co}$ radiation. ${ }^{21-23}$

The uncertainty associated with alanine dosimetry results was estimated following the recommendations of GUM. ${ }^{24}$ The type A uncertainty component was estimated from the standard deviation of the mean dose of the five irradiated pellets and includes the reproducibility of the measured dose, the uncertainty of the delivered MU, and the reproducibility of the motorized mechanism of the phantom in positioning the pellets at the reference depth. The type B component includes the uncertainty associated with the calibration procedure of the alanine pellets in the ${ }^{60} \mathrm{Co}$ beam, the difference in alanine response in the ${ }^{60} \mathrm{Co}$ and the MV beams, the correction for influence quantities (i.e., dose received during transport and signal fading), and the establishment of the reference conditions (i.e., $50 \mathrm{~mm}$ depth, $\mathrm{SSD}=750 \mathrm{~mm}$ ). The uncertainty in the establishment of the reference conditions is related to the dose uncertainty via the gradient of the depth dose distribution ${ }^{25}$ and was calculated using: (a) A standard uncertainty in determining the SSD of $u_{\mathrm{SSD}}=0.29 \mathrm{~mm}$ (calculated assuming rectangular distribution and an uncertainty of $\pm 0.5 \mathrm{~mm}$ in positioning the $\mathrm{x}$-ray source) and (b) a standard uncertainty in defining the reference depth of $u_{\text {depth }}=0.12 \mathrm{~mm}$ (calculated assuming rectangular distribution and an uncertainty of $\pm 0.2 \mathrm{~mm}$ in determining the reference depth).

\section{II.B.3. Ion chamber measurements}

One aim of this work was the experimental determination of the $k_{Q_{\text {msr }}}^{f_{\text {msr }}, f_{\text {ref }}}$ factor of a Farmer type ion chamber for measurements in the $60 \mathrm{~mm}$ diameter $f_{\mathrm{msr}}$ of the CyberKnife System. The PTW 30013 Farmer chamber was used, with 23 $\mathrm{mm}$ cavity length and $6.1 \mathrm{~mm}$ cavity diameter. A similar procedure to that described in Sec. II B 2 was used to align the chamber with the radiation beam axis. The chamber was used with its stem perpendicular to beam axis and the geometrical center of the cavity was placed at $50 \mathrm{~mm}$ depth in water. The $\mathrm{x}$-ray source was positioned at $800 \mathrm{~mm}$ SDD and $1106 \mathrm{MU}$ were delivered to exclude output linearity effects. Charge was measured using a PTW UNIDOS electrometer. A series of Farmer measurements were acquired immediately before and after the alanine irradiation and these results were averaged. Absorbed dose to water was calculated from these measurements using the TRS 398 formalism [i.e., excluding the new correction factor from Eq. (1)]. The $k_{Q_{\text {mrr }}}^{f_{\text {mr }}, f_{\text {ref }}}$ for the Farmer chamber was therefore obtained by dividing the Farmer dose measurement into the alanine dose measurements.

To assess the volume averaging effect, further measurements were performed using chambers with shorter cavity lengths. Specifically, the PTW 31010 and 31013 semiflex, PTW 31014 PinPoint, the CC13 (IBA Dosimetry GmbH, Germany), and the Exradin A16 (Standard Imaging, Inc. WI) ion chambers, with cavity lengths varying from 16.25 down to $2.7 \mathrm{~mm}$ were used. The measurement technique was identical to that described previously with the exception that a smaller MU setting of $150 \mathrm{MU}$ was used for each irradiation, and Farmer measurements were also repeated at this setting. Each measurement was repeated five times to enable statistical analysis.

All ion chambers and the electrometer were calibrated prior dosimetry measurements at the Ionizing Radiation Calibration Laboratory (IRCL) of the Greek Atomic Energy Commission (GAEC). The IRCL is traceable to the BIPM primary standard dosimetry laboratory. Calibration was performed in terms of absorbed dose to water $N_{D, w}$, using a ${ }^{60} \mathrm{Co}$ photon beam.

Beam quality correction factors $k_{Q, Q o}$ for all ion chambers were selected using linear interpolation of the data given in TRS-398 CoP (Ref. 14) except for the Exradin A16 which is not included in that report. The A16 $k_{Q_{Q} Q_{o}}$ value was calculated using the data of Kawachi et al. ${ }^{i 0}$ and the measured $\mathrm{TPR}_{20 / 10}\left(f_{\mathrm{msr}}\right)$ at $800 \mathrm{~mm}$ SDD. It should be noted that comparison of $k_{Q, Q_{o}}$ data for ion chambers with the same wall material (C552) used in the A16 chamber that are included in both TRS-398 (Ref. 14) and in Kawachi et al. ${ }^{10}$ showed excellent agreement $(0.1 \%)$.

The uncertainty associated with the chamber dose results was obtained using error propagation on the standard deviation of the mean charge measured by the electrometer (type A component) and on the type B components of: (a) The uncertainty of the calibration coefficient $N_{D, w}$ of the chamber; (b) the uncertainty associated with the establishment of the reference conditions (see also Sec. II B 2); (c) the uncer- 
tainty of the factors applied to correct the measured charge for polarity, ion recombination, temperature and pressure, etc.; and (d) the uncertainty associated with the beam quality correction factors. ${ }^{14}$ It is noted that the type B uncertainty components that affect all ion chamber results in a constant manner were excluded from the estimation of the composite uncertainty characterizing the comparison of the dosimetry results of the Farmer and the shorter cavity length cylindrical ion chambers. Specifically, these involved a) the traceability of the IRCL to the BIPM primary standard, b) the influence quantities (i.e., $k_{\mathrm{TP}}, k_{\text {elec }}$, and the component of the $k_{s}$ associated with the accuracy of the quadratic fit of the twovoltage method ${ }^{14}$ ) and c) the beam quality correction factor (only the components of $s_{w \text {,air }}$ and its assignment to beam quality and of the $W_{\text {air }} / e$ relative to ${ }^{60} \mathrm{Co}$ [see Table B.III in TRS398 CoP (Ref. 14)].

\section{II.C. Output factor measurements for small fields}

The suggested dosimetric formalism (see Sec. II A) was applied to measure the output factors $\Omega_{Q_{\mathrm{clin}}, Q_{\mathrm{msr}}}^{f_{\mathrm{clin}} f_{\mathrm{msr}}}$ of the $5,7.5$, 10 , and $15 \mathrm{~mm}$ diameter fixed collimators using a variety of dosimeters. Two microchambers, three diode detectors, alanine dosimeters, TLD microcubes, and EBT Gafchromic films were used.

\section{II.C.1. Diode and ion chamber measurements}

The Exradin A16 and the PTW PinPoint 31014 microchambers, as well as the two $p$-type PTW diodes (i.e., the shielded 60008 and the unshielded 60012) and the $n$-type EDGE diode detector (SunNuclear Corp., FL) were used. All detectors were placed with their stem parallel to beam axis except from the EDGE detector, which was positioned with its stem perpendicular to beam axis (this orientation aligns the active layer to the beam in an equivalent manner as the other diode detectors because of the EDGE detector design). Detectors were aligned with the radiation beam center for each collimator using the scanning method described previously. The effective point of measurement was then placed at $800 \mathrm{~mm}$ from the source at $15 \mathrm{~mm}$ depth from the surface, corresponding to the point of maximum dose along the depth dose curve of the $60 \mathrm{~mm}$ beam. For the A16 and the PinPoint chambers, the effective point was assumed to lay 2.2 and 3.7 mm from their external tip, respectively. ${ }^{3}$

All measurements were performed by delivering $100 \mathrm{MU}$ and averaged over a series of five repeated runs. Charge was measured using a UNIDOS electrometer. For the two chambers, a polarity effect was observed $( \pm 1.1 \%$ with respect to average for the $5 \mathrm{~mm}$ field size with the A16 and $\pm 2.5 \%$ with the PinPoint), and therefore the measurements performed with positive and negative $( \pm 400 \mathrm{~V})$ polarity were averaged. The raw measured output factors for the 5, 7.5, and $10 \mathrm{~mm}$ beams were multiplied with corresponding $k_{Q_{\mathrm{clin}}, Q_{\mathrm{msr}}}^{f_{\mathrm{msr}}, f_{\mathrm{ms}}}$ correction factors calculated using the method published by Francescon et al. ${ }^{3,4,15}$

The uncertainty of the measured output factors was obtained using error propagation on the standard deviation of the mean of the five measurements acquired for each field size. For the microchambers, the standard deviation of the mean of the output factors measured with positive and negative polarity was also considered during uncertainty estimation. The uncertainty associated with the $k_{Q_{\text {clin }}, Q_{\text {msr }}}^{f_{\text {mlin }}, f_{\text {msr }}}$ correction factors was assumed equal to $0.3 \%$ (taken from the statistical uncertainty of the MC simulations performed by Francescon et al. $^{3,4}$ ) and was considered in determining the overall uncertainty of the corrected output factors.

\section{II.C.2. Alanine and TLD measurements}

Alanine output factor measurements were performed using alanine pellets from the NPL batch (see Sec. II B 2). Corresponding TLD measurements were performed using LiF TLD type 100 microcubes of $1 \times 1 \times 1 \mathrm{~mm}^{3}$ (Harshaw/ Bicron, Solon, $\mathrm{OH})$. The TLD cubes were sorted, groupannealed $\left(1 \mathrm{~h}\right.$ at $400{ }^{\circ} \mathrm{C}$, followed by $2 \mathrm{~h}$ at $\left.100{ }^{\circ} \mathrm{C}\right)$, and then subjected to a prereadout annealing $\left(10 \mathrm{~min}\right.$ at $\left.100{ }^{\circ} \mathrm{C}\right)$ after radiation exposure. The TLDs were calibrated using a $100 \times 100 \mathrm{~mm}^{2} 6 \mathrm{MV}$ photon field. A total of $25 \mathrm{TLD}$ cubes were selected from a batch of 100 TLDs having a signal reproducibility better than $2 \%$ and used for small field output factor measurements. TLD readout was achieved by measuring the light output from each TLD cube with a TLD reader system (Model 2800M, Victoreen, Victoreen, Inc., Cleveland, $\mathrm{OH}$ ).

Both dosimeters were positioned with their effective point of measurement at the radiation beam center and $15 \mathrm{~mm}$ depth in water. This point was assumed to coincide with the geometrical center of each detector, which introduced a systematic uncertainty in the output factor results since both TLD and alanine are slightly denser than water. However, this uncertainty is less than $0.1 \%$ and $0.3 \%$ for the alanine and TLD measured output factors results, respectively, calculated based on the depth dose curves of the measured fields. Detector positioning for the alanine pellets has been described in Sec. II B 2, and was achieved for the TLD cubes in the same manner using a similar solid water adapter for the TRUFIX device (see Fig. 2, the cavity at the tip of the TLD adapter had a diameter of $1.5 \mathrm{~mm}$ and a depth of 1 $\mathrm{mm})$. A watertight sleeve of $0.1 \mathrm{~mm}$ thickness was used to shield these detectors from water during irradiation. Five alanine pellets and five TLD microcubes were irradiated for each field size. A nominal dose of 10 and $1 \mathrm{~Gy}$ was delivered to each alanine pellet and TLD microcube, respectively. Output factors were calculated as the ratio of the measured dose per MU of each field normalized to the corresponding ratio for the $60 \mathrm{~mm}$ reference field.

While the $5 \mathrm{~mm}$ alanine pellet diameter introduced no significant volume averaging effect in the $60 \mathrm{~mm}$ diameter msr field, this effect does become large for measurements of the small field sizes. Therefore, appropriate correction factors should be applied to the alanine measured output factors for the 5, 7.5, and $10 \mathrm{~mm}$ diameter beams. These were calculated by exploiting previously published small field dosimetry measurements performed using polymer gel water equivalent detectors. ${ }^{6}$ In more detail, the methodology described by Moutsatsos et al. ${ }^{26}$ was applied to the 3D dose 
distributions of each field size to calculate the average dose over all voxels lying within a cylinder of diameter $d$, and 2.5 $\mathrm{mm}$ height centered on the central voxel of the radiation beam, normalized to the average dose over the five voxels lying along the axis of the cylinder (the voxel size was 0.5 $\mathrm{mm}$, and therefore five voxels are contained within the 2.5 $\mathrm{mm}$ cylinder height). This procedure was performed for different cylinder diameters, using two irradiated gel vials and results were averaged. From these data, volume averaging correction factors as a function of detector diameter and field size were obtained and applied to the alanine output factor measurements.

The uncertainty assigned to the output factors measured using the alanine and TLD detectors was calculated using error propagation on the standard deviation of the mean of the five detectors irradiated for each field size and the uncertainty associated with the calibration procedure. The systematic uncertainty associated with assuming the effective point of measurement to coincide with the geometric center of each detector was included in the TLD measurement uncertainty, but omitted from the alanine measurement uncertainty because of its small magnitude $(<0.1 \%)$. The uncertainty associated with the volume average correction factors was also considered for determining the overall uncertainty of the alanine output factors.

\section{II.C.3. Gafchromic EBT film measurements}

Output factors were also measured using Gafchromic EBT films (ISP, Wayne, NJ). Film dosimetry data acquisition and processing was performed following the protocol suggested by Devic et al. ${ }^{27}$ Output factor measurements were performed by placing precut EBT films (Lot \#: 47277-03I) of $60 \times 60 \mathrm{~mm}^{2}$ size at $15 \mathrm{~mm}$ depth in RW3 solid water slabs of $200 \mathrm{~mm}$ total thickness and $300 \times 300 \mathrm{~mm}^{2}$ in plane dimensions. The x-ray source was placed $800 \mathrm{~mm}$ from the film surface. Five films were irradiated serially for each field size to a nominal dose of 500 cGy. All EBT films were scanned one day postirradiation to allow postirradiation optical density growth, using an Epson Expression 1680Pro flatbed optical scanner. The Epson scanner was used in transmission mode and all films were scanned in 48-bit RGB mode with a resolution of $150 \mathrm{dpi}$ (pixel size $=0.169 \mathrm{~mm}$ ), but only the red color channel of the image was used.

The symmetry of the radiation field depicted on the film's plane was used to define the coordinates of the field center. ${ }^{6}$ The pixel values lying within a circle of $0.5 \mathrm{~mm}$ diameter for the small fields and $5 \mathrm{~mm}$ diameter for the $60 \mathrm{~mm}$ field, centered at the corresponding radiation field center, were averaged and used to calculate the optical density (OD) of each film. The OD values of the films irradiated with the same field were averaged and converted to dose using the calibration curve of the film batch used in this work. Output factors were calculated as the ratio of the measured dose per MU of each field normalized to the corresponding ratio for the 60 $\mathrm{mm}$ reference field. The uncertainty of the measured output factors was obtained using error propagation on the uncertainty of the measured dose at the center of each field. The
TABLE I. Alanine and Farmer (PTW 30013) chamber calibration dosimetry results. The yielded $k_{Q_{\mathrm{msr}},}^{f_{\mathrm{ms}} f_{\mathrm{r}}}$ factor of the Farmer chamber is also presented.

\begin{tabular}{lcc}
\hline \hline & \multicolumn{2}{c}{ Detector } \\
\cline { 2 - 3 } Parameter & Alanine & Farmer \\
\hline Calibration laboratory & $\mathrm{NPL}$ & $\mathrm{IRCL}^{\mathrm{a}}$ \\
Calibration beam quality & ${ }^{60} \mathrm{Co}$ & ${ }^{60} \mathrm{Co}$ \\
$N_{D, w}(\mathrm{cGy} / \mathrm{nC})$ & $\ldots$ & 5.41 \\
Beam quality correction, $k_{Q, Q o}$ & 1.006 & 0.992 \\
Delivered MUs & 1106 & 1106 \\
Average measured charge (nC) & $\ldots$ & 180.71 \\
Correction for influence quantities & \multicolumn{2}{c}{} \\
Polarity, $k_{\text {pol }}$ & $\ldots$ & 1.0015 \\
Ion recombination, $k_{s}$ & $\ldots$ & 1.003 \\
Temperature-pressure, $k_{\mathrm{TP}}$ & $\ldots$ & 1.030 \\
Electrometer calibration, $k_{\text {elec }}$ & $\ldots$ & 0.998 \\
Measured dose (cGy) & 1000.8 & 1001.4 \\
$k_{Q_{\text {msr }} f_{\text {ref }}}$ & \multicolumn{2}{c}{0.999} \\
\hline \hline
\end{tabular}

${ }^{\mathrm{a}}$ Ionizing Radiation Calibration Laboratory (GAEC); traceable to BIPM.

uncertainty associated with the dose corresponding to the center of each field was determined using error propagation on the standard deviation of the mean OD and the uncertainty associated with the calibration procedure.

\section{RESULTS}

\section{III.A. Reference dosimetry in the msr field}

$\operatorname{TPR}_{20 / 10}\left(f_{\mathrm{msr}}\right)$ index values of 0.639 and 0.648 were measured in the msr field with SDD of 800 and $1000 \mathrm{~mm}$, respectively. Converting these values to the hypothetical $100 \times 100 \mathrm{~mm}^{2}$ reference field gave a $\mathrm{TPR}_{20 / 10}$ value of 0.664 using the Sauer ${ }^{19}$ function and 0.666 using the BJR supplement 25 (Ref. 20) data tables. Both beam quality index values agree within $0.3 \%$ confirming the small dependence of TPR on the measurement distance and the equivalence of these conversion methods. This difference has no influence $(\sim 0.03 \%)$ on the $k_{Q, Q o}$ value and, therefore, the beam quality value of 0.666 corresponding to the recommended $1000 \mathrm{~mm}$ SDD in the TRS-398 CoP (Ref. 14) was used to select $k_{Q, Q o}$ values for the ion chambers employed for reference dosimetry measurements in this work.

Table I presents calibration dosimetry details using the alanine pellets and the Farmer ion chamber. The corresponding uncertainties associated with the calibration dosimetry measurements are presented in Table II. Doses of $1000.8 \pm 10.7$ and $1001.4 \pm 12.5$ cGy were measured, respectively. In terms of the proposed dosimetric formalism, this result shows that a Farmer chamber can be used for the msr calibration measurement, with $k_{Q_{\text {msr }}}^{f_{\text {mse }}} f_{\text {ref }}=0.999 \pm 0.016$. It is noted that the uncertainty of this correction factor is relatively large and its largest single component is the $k_{Q, Q o}$ factor uncertainty (see Table II).

To refine the correction factor for the Farmer chamber, reference dosimetry results of this specific chamber were compared to corresponding results obtained using ion chambers with shorter cavity lengths. These results are presented 
TABLE II. Estimated uncertainties associated with the alanine and Farmer chamber calibration dosimetry results expressed at $68 \%$ confidence level. The corresponding uncertainty of the $k_{Q_{\mathrm{msr}},}^{f_{\mathrm{ms}} \text { ref }}$ factor is also presented.

\begin{tabular}{|c|c|c|c|c|}
\hline \multirow[b]{2}{*}{ Component } & \multicolumn{2}{|c|}{ Alanine } & \multicolumn{2}{|c|}{ Farmer } \\
\hline & $\begin{array}{c}\text { Type A } \\
(\%)\end{array}$ & $\begin{array}{c}\text { Type B } \\
(\%)\end{array}$ & $\begin{array}{c}\text { Type A } \\
(\%)\end{array}$ & $\begin{array}{c}\text { Type B } \\
(\%)\end{array}$ \\
\hline Reproducibility of the reading & 0.61 & $\cdots$ & 0.26 & .. \\
\hline Calibration procedure & $\cdots$ & 0.68 & $\cdots$ & 0.55 \\
\hline Establishment of reference conditions & $\cdots$ & 0.10 & $\cdots$ & 0.10 \\
\hline Correction for influence quantities & $\ldots$ & 0.28 & $\cdots$ & $0.40^{\mathrm{a}}$ \\
\hline Beam quality, $k_{Q, Q o}$ & $\cdots$ & 0.35 & $\cdots$ & $1.00^{\mathrm{a}}$ \\
\hline Combined uncertainty & \multicolumn{2}{|c|}{1.02} & \multicolumn{2}{|c|}{1.24} \\
\hline$k_{Q_{\mathrm{msr}}}^{f_{\mathrm{ms}} f_{\text {ref }}} Q$ & \multicolumn{4}{|c|}{1.61} \\
\hline
\end{tabular}

${ }^{\mathrm{a}}$ Taken from the TRS-398 code of practice (Table VI.IV) (Ref. 14).

in Fig. 3 normalized to the Farmer chamber measurement. Although all measurements agree within experimental uncertainties, they follow a trend of increasing mean dose measurement with decreasing cavity length. It should be noted that in terms of the suggested dosimetric formalism [Eq. (1), Sec. II A], the ratio of the measured chamber dose results corresponds to the ratio of the $k_{Q_{\text {msrr }}}^{f_{\text {ms }},}$ ref $_{\text {ref }}$ factor values. In Fig. 3, calculated $k_{Q_{\text {ms }}}^{f_{\text {msr }}, f_{\text {ref }}}$ factor values taking into account only the volume averaging contribution [obtained using Eq. 2 in Ref. 10] of the radiation dose profile at $50 \mathrm{~mm}$ depth (see Fig. 1), for a chamber with a $6 \mathrm{~mm}$ constant cavity diameter are plotted versus cavity length $l$, normalized to the corresponding factor for a cavity length of $23 \mathrm{~mm}$. Corresponding calculations were also performed for the actual cavity lengths and diameters of the cylindrical ion chambers used in this work and the results are presented in Fig. 3 to aid comparison. These volume averaging corrections show a similar trend to the measured data.

\section{III.B. Output factors for small fields}

The ratio of detector readings in the $f_{\text {clin }}$ and $f_{\text {msr }}$, (i.e., the uncorrected output factors) for the 5, 7.5, and $10 \mathrm{~mm}$ small fields are presented in Table III and graphically in Fig. 4(a).

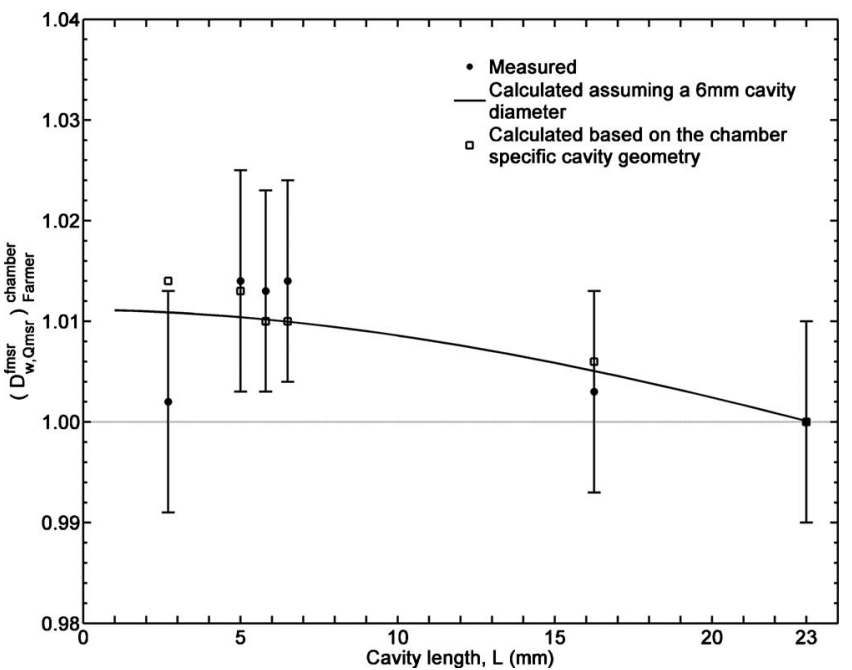

FIG. 3. Measured absorbed dose to water $D_{w, Q_{\text {msr }}}^{f_{\text {msr }}}$ for the $60 \mathrm{~mm}$ fixed collimator msr field at $800 \mathrm{~mm}$ SDD using ion chambers of different cavity length. All results are normalized to the corresponding result of the Farmer chamber. Presented uncertainties correspond to the $68 \%$ confidence level. Estimated measurement ratios taking into account only the volume average effect are also shown for a chamber with cavity diameter of $6 \mathrm{~mm}$ and variable length (solid line) as well as for the specific cavity dimensions of the chambers used in this study (open square).

Corresponding polymer gel values previously published by our group ${ }^{6}$ are also included for comparison purposes. A general inspection of the presented data confirms the findings of other authors that the deviations between output factors measured using different detectors are substantial for small fields, and increase with decreasing field size. . $^{3-7,28-31}$

The correction factors $k_{Q_{\text {clin }}, Q_{\text {msr }}}^{f_{\text {clin }} f_{\text {msr }}}$ calculated using the method of Francescon et al. ${ }^{3,4,15}$ for the two microchambers (i.e., the Exradin A16 and the PinPoint 31014) and the three diode detectors (i.e., the PTW 60008 and 60012 and the EDGE detector) are presented in Table III. Volume average correction factors calculated using the polymer gels (see Sec. II C 2) for the 5, 7.5, and $10 \mathrm{~mm}$ clinical field sizes are presented in Fig. 5. Error weighted least square regression was performed on the presented data sets and the volume

TABLE III. Ratio of detector readings $M_{\mathcal{Q}_{\text {clin }}}^{f_{\text {lin }}} / M_{Q_{\text {msr }}}^{f_{\text {msr }}}$ and correction factors $k_{Q_{\text {clin }} \mathcal{Q}_{\text {msr }}}^{f_{\text {lin }} f_{\text {sr }}}$ for the $5,7.5$, and $10 \mathrm{~mm}$ fields. Corresponding uncertainties at $68 \%$ level are shown in parentheses, indicating the uncertainty in the final one or two digits.

\begin{tabular}{|c|c|c|c|c|c|c|}
\hline \multirow[b]{2}{*}{ Detector } & \multicolumn{2}{|c|}{$5 \mathrm{~mm}$} & \multicolumn{2}{|c|}{$7.5 \mathrm{~mm}$} & \multicolumn{2}{|c|}{$10 \mathrm{~mm}$} \\
\hline & $M_{Q_{\mathrm{clin}}}^{f_{\mathrm{clin}}} / M_{Q_{\mathrm{msr}}}^{f_{\mathrm{msr}}}$ & $\begin{array}{l}k_{Q_{\mathrm{clin}}, Q_{\mathrm{msr}}}^{f_{\mathrm{clin}}, f_{\mathrm{msr}}} \\
\end{array}$ & $M_{Q_{\mathrm{clin}}}^{f_{\mathrm{clin}}} / M_{Q_{\mathrm{msr}}}^{f_{\mathrm{msr}}}$ & $\begin{array}{l}k_{Q_{\mathrm{clin}},}^{f_{\mathrm{clin}}, f_{\mathrm{msr}}}, Q_{\mathrm{msr}} \\
\end{array}$ & $M_{Q_{\mathrm{clin}}}^{f_{\mathrm{clin}}} / M_{Q_{\mathrm{msr}}}^{f_{\mathrm{msr}}}$ & $\begin{array}{l}k_{Q_{\mathrm{clin}}, Q_{\mathrm{msr}}}^{f_{\mathrm{clin}}, f_{\mathrm{msr}}} \\
\end{array}$ \\
\hline A16 & $0.626(15)$ & 1.089 (3) & $0.811 \quad(10)$ & 1.018 (3) & $0.866(6)$ & $1.010(3)$ \\
\hline PinPoint & $0.620(17)$ & $1.101(3)$ & $0.801 \quad(7)$ & $1.024(3)$ & $0.862(5)$ & 1.015 (3) \\
\hline Diode 60008 & $0.726(1)$ & $0.943(3)$ & $0.873(1)$ & 0.949 (3) & $0.912(1)$ & 0.964 (3) \\
\hline Diode 60012 & $0.705(1)$ & $0.956(3)$ & $0.847(2)$ & 0.966 (3) & $0.891 \quad(1)$ & 0.978 (3) \\
\hline EDGE & $0.726(1)$ & 0.948 (3) & 0.864 (1) & 0.955 (3) & $0.906(1)$ & $0.966(3)$ \\
\hline Alanine & $0.544(8)$ & $1.249(8)$ & $0.785(12)$ & 1.059 (4) & 0.855 (13) & 1.019 (3) \\
\hline TLD & 0.668 & $\cdots$ & $0.809(6)$ & $\cdots$ & $0.880(8)$ & $\cdots$ \\
\hline EBT films & 0.659 (17) & $\ldots$ & $0.811(16)$ & $\cdots$ & 0.853 (18) & $\ldots$ \\
\hline Polymer gels ${ }^{\mathrm{a}}$ & $0.702(21)$ & $\cdots$ & $0.872(27)$ & $\cdots$ & 0.929 (29) & $\cdots$ \\
\hline
\end{tabular}

${ }^{\mathrm{a}}$ Reference 6 . 

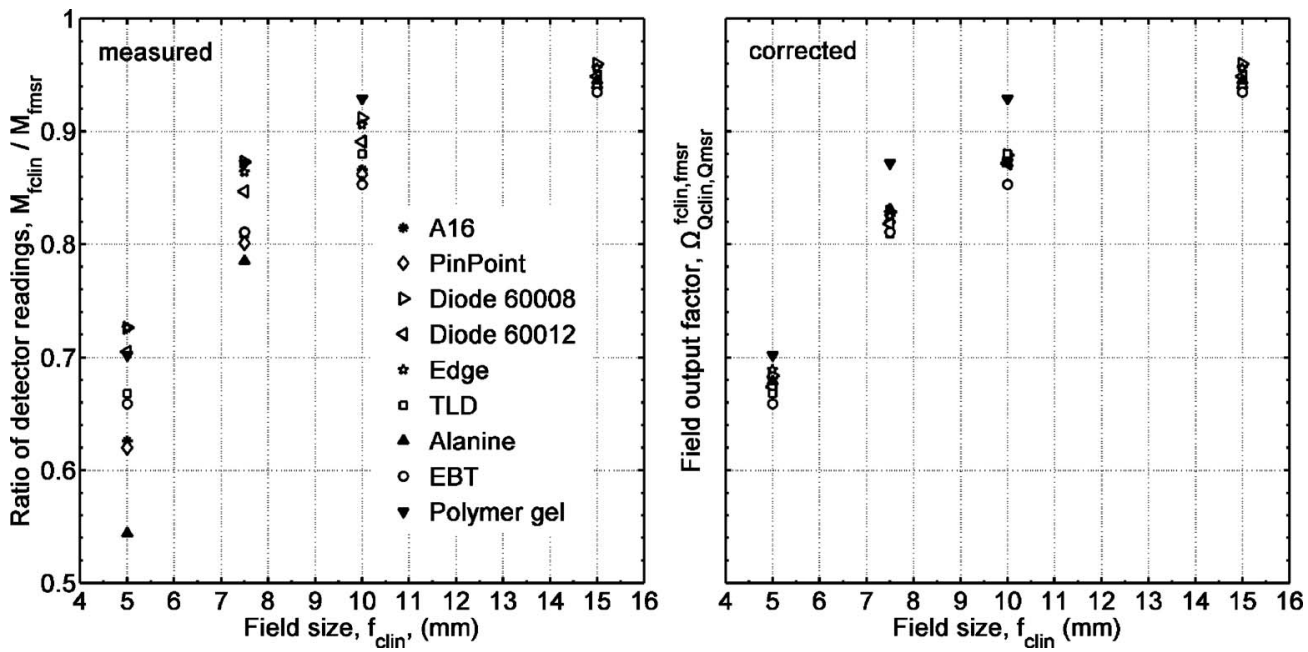

FIG. 4. Output factors of the 5, 7.5, 10, and $15 \mathrm{~mm}$ diameter fixed collimators at $800 \mathrm{~mm}$ SDD (a) before and (b) after correction factors $k_{Q_{\text {clinim }} Q_{\mathrm{msr}}}^{f_{\text {mil }}, f_{\mathrm{ms}}}$ were applied to the microchamber, diode detector, and alanine dosimeter measurements.

average correction factors for the alanine pellet dimensions were found equal to $1.249 \pm 0.008,1.059 \pm 0.003$, and $1.019 \pm 0.003$ for the $5,7.5$, and $10 \mathrm{~mm}$ clinical fields, respectively.

The corrected output factors $\Omega_{Q_{\text {clin }}, Q_{\text {msr }}}^{f_{\text {cin }}, f_{\text {mr }}}$ of the $5,7.5$, and $10 \mathrm{~mm}$ clinical fields are presented in Table IV and plotted in Fig. 4(b) along with corresponding TLD and EBT results as well as previously published polymer gel values. ${ }^{6}$ The uncorrected output factors of the $15 \mathrm{~mm}$ field are also presented in Fig. 4 and Table IV for completeness. The deviations observed between measured output factors decrease substantially after applying the correction factors. The weighted mean of the corrected output factors were found equal to $0.681 \pm 0.001, \quad 0.824 \pm 0.001, \quad 0.875 \pm 0.001, \quad$ and

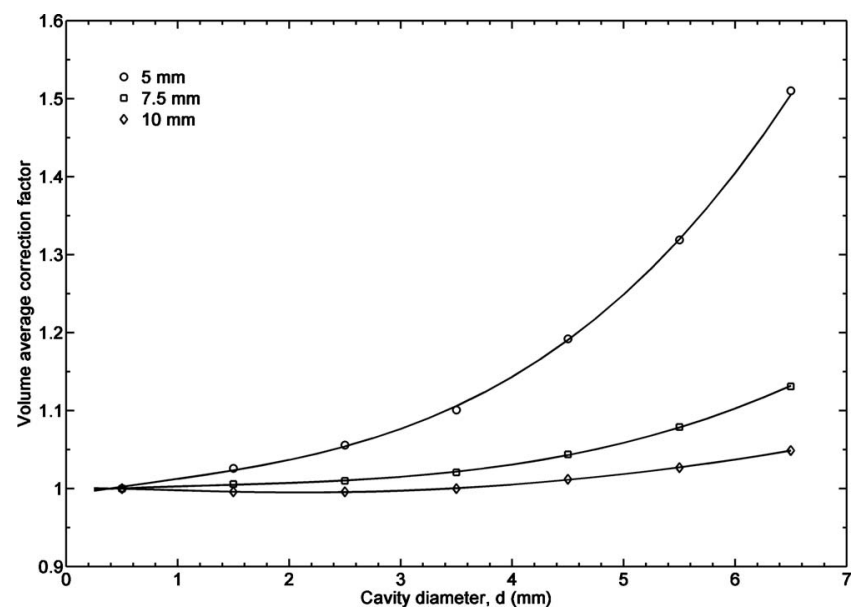

FIG. 5. Volume averaging correction factors, calculated using 3D polymer gel dosimetry results (Ref. 6) and assuming cylindrical detectors of $2.5 \mathrm{~mm}$ height and varying cavity diameter for the $5,7.5$, and $10 \mathrm{~mm}$ fixed collimator beams at $800 \mathrm{~mm}$ SDD. The uncertainty of the presented results is of the order of $4 \%$ and the solid lines correspond to weighted least square regression fit results. In the absence of all other measurement perturbations, these data correspond to $k_{Q_{\text {clin }}, Q_{\text {msr }}}^{f_{\text {clin }}, f_{\text {msr }}}$ values.
$0.954 \pm 0.001$ for the $5,7.5,10$, and $15 \mathrm{~mm}$ beams, respectively, forming a consensus output factor data set for the CyberKnife System used in this study.

\section{DISCUSSION}

The $k_{Q_{\mathrm{msr}},}^{f_{\mathrm{ms}},}, f_{\text {ref }}$ correction factor of the Farmer chamber was found equal to $0.999 \pm 0.016$. This result is in agreement with the $k_{Q_{\mathrm{msr}}}^{f_{\mathrm{mrr}},}, f_{\text {ref }}$ values of 0.999 and 0.996 reported by Alfonso et al. ${ }^{13}$ However, it should be noted that these values were determined using previously published MC simulation results ${ }^{8,9}$ that investigated only the differences in the beam quality correction factors between the $f_{\mathrm{msr}}$ and $f_{\text {ref }}$ fields, ignoring the effect of dose gradient in the detector geometry. These values are similar to the finding that $k_{Q, Q o}$ is overestimated by about $0.5 \%$ (i.e., the $k_{Q_{\mathrm{msr}},}^{f_{\mathrm{mrr}}, f_{\text {ref }}}$ is $\sim 0.995$ assuming only the beam quality component) when $\mathrm{TPR}_{20 / 10}$ is used as an energy specifier for $6 \mathrm{MV}$ flattening filter free beams. $^{32}$

TABLE IV. Output factors $\Omega_{Q_{\text {clin, }}, Q_{\text {msr }}}^{f_{\text {lin }} f_{\text {sr }}}$ for the $5,7.5,10$, and $15 \mathrm{~mm}$ fields, after correction factors are applied at all field sizes except $15 \mathrm{~mm}$, as shown in Table III. Corresponding uncertainties at $68 \%$ level are shown in parentheses indicating the uncertainty in the final one or two digits.

\begin{tabular}{|c|c|c|c|c|}
\hline Detector & $5 \mathrm{~mm}$ & $7.5 \mathrm{~mm}$ & $10 \mathrm{~mm}$ & $15 \mathrm{~mm}$ \\
\hline A16 & $0.682(17)$ & 0.825 & $0.874(7)$ & 0.939 \\
\hline PinPoint & $0.683(18)$ & $0.820(8)$ & 0.875 & $0.939(2)$ \\
\hline Diode 60008 & 0.684 (2) & 0.829 & 0.879 (3) & 0.960 \\
\hline Diode 60012 & $0.674(2)$ & 0.818 & 0.872 & 0.949 (1) \\
\hline EDGE & $0.689(2)$ & 0.825 & 0.875 & 0.956 \\
\hline Alanine & 0.679 (11) & 0.831 & $0.872(13)$ & 0.945 (14) \\
\hline TLD & 0.668 & $0.809(6)$ & $0.880(8)$ & $0.941(7)$ \\
\hline EBT films & 0.659 (17) & 0.811 & 0.853 & $0.935(20)$ \\
\hline Polymer gels ${ }^{\mathrm{a}}$ & $0.702(21)$ & $0.872(27)$ & 0.929 (29) & $\ldots$ \\
\hline Weighted mean & 0.681 & 0.824 & 0.875 & 0.954 \\
\hline
\end{tabular}

${ }^{\mathrm{a}}$ Reference 6. 
On the other hand, comparison of the Farmer calibration dosimetry results against corresponding results using ion chambers with shorter cavity lengths revealed that, higher dose measurements are obtained with smaller cavity dimensions. A maximum difference of $1.4 \%$ is observed with the PinPoint 31014 and the Semiflex 31010 chambers, with cavity lengths of 5 and $6.5 \mathrm{~mm}$, respectively. These differences are in agreement $(\leq 0.3 \%)$ with those predicted by theoretical volume averaging calculations based on the msr beam profile across the chamber cavity (see Figs. 1 and 3), suggesting that the Farmer $k_{Q_{\mathrm{msr}}}^{f_{\mathrm{msr}}, \mathrm{f}_{\mathrm{ref}}}$ correction factor assuming only the volume averaging contribution is equal to 1.014.

Combining the contributions of the beam quality and volume averaging components on the Farmer correction factor shows that a value of $k_{Q_{\text {msr }}}^{f_{\text {msr }},} f_{\text {ref }}=1.01$ could be reasonably assumed for measurements at $50 \mathrm{~mm}$ depth. This agrees within measurement uncertainty with the result of $k_{Q_{\text {msr }}}^{f_{\text {msr }} f_{\text {ref }}}=0.999 \pm 0.016$ obtained by comparison with alanine dosimetry. However, because of the relatively large measurement uncertainties this value requires further validation using MC simulations.

Alternatively, ion chambers with shorter cavity lengths have been suggested for CyberKnife calibration purposes. ${ }^{10}$ For example, shortened Farmer chambers with a cavity length of $\sim 9 \mathrm{~mm}$ could be used. According to the data presented in Fig. 3, chambers with cavity length less than 10 $\mathrm{mm}$ exhibit minimal volume averaging effects. It should be noted, however, that chambers with very small cavity dimensions may introduce other measurement uncertainties (e.g., in this work the Exradin A16 presented abnormal saturation effects, i.e., ionization chamber signal increased with decreasing applied voltage) in agreement with findings published in the literature, ${ }^{12,33,34}$ which might pose a limitation for their use in the msr field calibration.

Regarding output factor results, the $k_{Q_{\text {clin }}, Q_{\text {msr }}}^{f_{\text {cin }}, f_{\text {ms }}}$ correction factors applied to 60008 diode measurements were calculated as $0.943,0.949$, and 0.964 for the $5,7.5$, and $10 \mathrm{~mm}$ fields, respectively, suggesting an overestimation in the measured output factors of $5.7 \%, 5.1 \%$, and $3.6 \%$. Similar $k_{\mathcal{Q}_{\text {clin }}, Q_{\text {msr }}}^{f_{\text {clin }}, f_{\text {msr }}}$ factors were also obtained for the unshielded 60012 diode and the copper shielded EDGE detector. The unshielded diode presented the smaller overestimation of $4.4 \%$, $3.4 \%$, and $2.2 \%$ for the $5,7.5$, and $10 \mathrm{~mm}$ fields, respectively. $k_{Q_{\text {clin }}, Q_{\text {msr }}}^{f_{\text {min }}, f_{\text {ms }}}$ factors below unity for diode detectors have been attributed to the perturbation in the local particle fluence caused by the presence of the small but relatively dense $\left(\rho=2.33 \mathrm{~g} \mathrm{~cm}^{-3}\right)$ silicon detector in the field. ${ }^{17}$ This perturbation is higher in the case of tungsten or copper high atomic number materials used as backing mediums in the shielded 60008 diode and the EDGE detector, respectively, and explains the smaller $k_{Q_{\text {clin }}, Q_{\text {msr }}}^{f_{\text {lin }}, f_{\text {sr }}}$ value obtained for the unshielded 60012 diode.

The $k_{Q_{\text {clin }}, Q_{\text {msr }}}^{f_{\text {min }}, f_{\text {msr }}}$ correction factors taking into account the volume averaging effect, for the alanine measured output factors were found equal to $1.249,1.059$, and 1.019 for the 5 , 7.5 , and $10 \mathrm{~mm}$ fields, respectively. These greater than unity $k_{Q_{\text {clin }}, Q_{\text {msr }}}^{f_{\text {lin }}, f_{\text {sr }}}$ values are attributed to dose averaging over the sensitive volume of the alanine pellets; the pellet diameter was equal to the nominal FWHM of the smallest beam size available (i.e., $5 \mathrm{~mm}$ ). Greater than unity $k_{Q_{\text {clin }}, Q_{\text {msr }}}^{f_{\text {lin }}, f_{\text {msr }}}$ factors were also obtained for the A16 and PinPoint small cavity ion chambers. Despite their small dimensions, these $k_{Q_{\text {clin }}, Q \text { msr }}^{f_{\text {ms }}}$ values suggest an underestimation of up to $9 \%$ and $10 \%$ for the $5 \mathrm{~mm}$ field for the A16 and the PinPoint chambers, respectively. These corrections are generally attributed to volume average effects. ${ }^{2,5,7}$ It is worth noting, however, that according to the data of Fig. 5, the volume effect corrections for cavity diameters of 2.4 (A16) and $2 \mathrm{~mm}$ (PinPoint) are of the order of just $5 \%$ and $4 \%$, respectively, for the $5 \mathrm{~mm}$ field. Corresponding volume averaging corrections for the $7.5 \mathrm{~mm}$ field are reduced to $1 \%$ for the A16 and $0.7 \%$ for the PinPoint, compared to $k_{Q_{\text {clin }}, Q_{\text {msr }}}^{f_{\text {ms }}, f_{\text {mal }}}$ values suggesting an underestimation of $1.8 \%$ and $2.4 \%$ for the same chambers. These findings suggest that the observed measurement underestimation with the A16 and PinPoint chambers is only partly attributed to the volume averaging effect, and partly due to a change in the fluence perturbations caused by the presence of the detector in the small field compared to the msr field. ${ }^{35}$

The method developed by Francescon et al. ${ }^{3,4,15}$ and employed in this work for calculating correction factors for the microchamber and diode measured output factors combines both MC simulations and measurements. It is based on the assumption that the response of each detector is the same in both simulation and measurement. Francescon et al. ${ }^{3,4}$ validated their method by comparing microchamber and diode measured output factors before and after correction factors were applied. A similar comparison was also performed in this work considering also output factor results generated using alanine, TLD, and EBT water equivalent dosimeters. For the $5 \mathrm{~mm}$ field, all microchamber and diode corrected output factors agree within $1.5 \%$ and $3 \%$ compared to alanine and TLD results, respectively. An agreement within 5\% can also be observed in comparison with the EBT measured values and the polymer gel published values, which is within the relatively increased uncertainties of these two methods. A similarly good agreement can also be observed for the 7.5 $\mathrm{mm}$ (all chamber and diode corrected output factors agree within $1.6 \%$ and $2.5 \%$ with the alanine and TLD results, respectively) and $10 \mathrm{~mm}$ fields (all chamber and diode corrected output factors agree within $0.8 \%$ and $1.0 \%$ with the alanine and TLD results, respectively), with only the polymer gel values deviating by more than one standard uncertainty $(1 \sigma)$ from the corrected and measured results of other detectors used in this study. This excellent agreement between corrected chamber and diode measurements and both alanine and TLD results provides an independent validation of the Francescon et al. ${ }^{3,4}$ method for calculating $k_{Q_{\text {clin }}, Q_{\text {msr }}}^{f_{\text {cin }} f_{\text {mr }}}$ factors. In combination with the large variations observed in measured output factors before correction (see Table III), these results support the application of $k_{Q_{\text {clin }}, Q_{\text {msr }}}^{f_{\text {lin }} f_{\text {sr }}}$ correction factors whenever small field output factor measurements are performed using diode detectors or microchambers.

Data processing performed to determine the $k_{Q_{\text {clin }} Q_{\text {msr }}}^{f_{\text {min }} f_{\text {msr }}}$ correction factor for each type of diode detector used in this 
work showed that this factor is essentially independent (within $0.6 \%$ ) of the focal spot size and, therefore, a constant $k_{Q_{\text {clin }}, Q_{\text {msr }}}^{f_{\text {lin }}, f_{\text {sr }}}$ can be used for a given diode design and field size. The correction factors for microchambers on the contrary, present a focal spot size dependent variation of up to $4 \%,{ }^{3}$ which, combined with the intense polarity effect observed, made the experimental procedure more complex and errorprone. As a result, chamber measured output factors are associated with higher levels of uncertainty compared to diodes. At this point, it should be noted that, the uncertainty of the $k_{Q_{\text {clin }}, Q_{\text {msr }}}^{f_{\text {cin }} f_{\text {ms }}}$ correction factors owing to the fitting routine suggested by Francescon et al. ${ }^{3,4}$ was not considered in total uncertainty estimation, since corresponding calculations resulted to artificially high uncertainty values ( $14 \%$ for the A16, $10 \%$ for the PinPoint and the $5 \mathrm{~mm}$ field), which were attributed to the limited number of points provided for the fitting process.

Finally, and in view of the above discussion, it is worth noting that for the $15 \mathrm{~mm}$ field, the uncorrected chamber results ( 0.939 for both chambers) are lower than all the diode results $(0.949,0.956$, and 0.960$)$. Both alanine and TLD dosimeters measured the same output factor value of 0.944 , which agree within uncertainties with the results obtained using the chambers and the unshielded diode. While these differences between uncorrected results are much lower than the corresponding differences observed in the smaller fields, a slight improvement in measurement accuracy would be achieved if the MC based method suggested by Francescon et $a l .{ }^{3,4,15}$ for calculating correction factor data was extended to the $15 \mathrm{~mm}$ beam.

\section{CONCLUSIONS}

A recently proposed formalism was applied for calibration dosimetry of the CyberKnife System, using a Farmer chamber (PTW 30013) and a series of cylindrical chambers of shorter cavity length. The Farmer response was compared to alanine dosimetry obtained using the therapy-level alaninedosimetry service provided by the NPL. The $k_{Q_{\text {msr }}}^{f_{\text {ms }}, f_{\text {ref }}}$ of the Farmer chamber was found equal to $0.999 \pm 0.016$. Comparison of the Farmer dose response against that of ion chambers with shorter cavity lengths showed that while results agree within experimental uncertainties, the mean measured values closely follow the trend expected by theoretical volume averaging calculations in the msr field, giving an underestimate in the Farmer reading of $1.4 \%$. Combining this result with findings of the literature on the expected overestimate of $\sim 0.5 \%$ in $k_{Q, Q o}$ for $6 \mathrm{MV}$ flattening filter free photon beams, suggests that a Farmer dose response correction of $k_{Q_{\text {msr }}}^{f_{\text {ms }}, f_{\text {ref }}}$ $=1.01$ would be consistent with theoretical expectation, which is also consistent within measurement uncertainty with the experimental finding using alanine dosimetry. Shorter cavity length chambers (less than $10 \mathrm{~mm}$ ) should have a smaller $k_{Q_{\text {msr }}}^{f_{\text {mse }}}, f_{\text {ref }}$ factor in the range $0.995-1.000$ due to the minimal volume average contribution and residual beam quality correction uncertainty.

Large variations in measured small field output factors were observed between small volume ion chambers, diode detectors, alanine pellets, radiochromic film, TLD, and polymer gel dosimetry. After the corrections described in the new dosimetry formalism were applied to the ion chamber and solid state detector measurements using the method of Francescon $e t a l .,{ }^{3,4,15}$ and to the alanine data using the volume averaging correction factors calculated in this work, the output factor agreement between all chambers and diode detectors was within $1.6 \%$ of the alanine result for the three smallest fields. The corrected output factors of the microchambers and diode detectors were found to agree within experimental uncertainties with corresponding results of this work using alanine dosimeters, TLD microcubes and EBT films.

\section{ACKNOWLEDGMENTS}

The work was partly supported by Accuray Inc. and the Ministry of National Education and Religious Affairs within the framework of the project "Irakleitos." The authors would like to acknowledge the contribution of Mu Young Lee, Accuray Inc., for loaning the Exradin A16 microchamber and the EDGE detector and for the helpful discussions. The contributions of Constantinos Hourdakis (IRCL/GAEC) in the ion chambers' calibration procedure and of Peter Sharpe (NPL) in alanine dosimetry are also gratefully acknowledged.

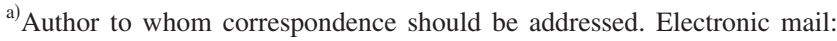
vpantelis@phys.uoa.gr

${ }^{1}$ I. J. Das, G. X. Ding, and A. Ahnesjo, "Small fields: Nonequilibrium radiation dosimetry," Med. Phys. 35, 206-215 (2008).

${ }^{2}$ J. Fan, K. Paskalev, L. Wang, L. Jin, J. Li, A. Eldeeb, and C. Ma, "Determination of output factors for stereotactic radiosurgery beams," Med. Phys. 36, 5292-5300 (2009).

${ }^{3}$ P. Francescon, S. Cora, and C. Cavedon, "Total scatter factors of small beams: A multidetector and Monte Carlo study," Med. Phys. 35, 504-513 (2008).

${ }^{4}$ P. Francescon, S. Cora, C. Cavedon, and P. Scalchi, "Application of a Monte Carlo-based method for total scatter factors of small beams to new solid state micro-detectors," J. Appl. Clin. Med. Phys. 10, 147-152 (2008).

${ }^{5}$ W. U. Laub and T. Wong, "The volume effect of detectors in the dosimetry of small fields used in IMRT," Med. Phys. 30, 341-347 (2003).

${ }^{6}$ E. Pantelis, C. Antypas, L. Petrokokkinos, P. Karaiskos, P. Papagiannis, M. Kozicki, E. Georgiou, L. Sakelliou, and I. Seimenis, "Dosimetric characterization of CyberKnife radiosurgical photon beams using polymer gels," Med. Phys. 35, 2312-2320 (2008).

${ }^{7}$ O. A. Sauer and J. Wilbert, "Measurement of output factors for small photon beams," Med. Phys. 34, 1983-1988 (2007).

${ }^{8}$ P. Francescon, S. Cora, C. Cavedon, P. Scalhi, and J. Stancanello, “CyberKnife dosimetric beam characteristics: Comparison between experimental results and Monte Carlo simulation," in Robotic Radiosurgery (CyberKnife Society, Sunnyvale, 2005), Vol. 1, pp. 271-280.

${ }^{9}$ F. Araki, "Monte Carlo study of a Cyberknife stereotactic radiosurgery system," Med. Phys. 33, 2955-2963 (2006)

${ }^{10}$ T. Kawachi, H. Saitoh, M. Inoue, T. Katayose, A. Myojoyama, and K. Hatano, "Reference dosimetry condition and beam quality correction factor for CyberKnife beam," Med. Phys. 35, 4591-4598 (2008).

${ }^{11}$ R. Jeraj, T. R. Mackie, J. Balog, and G. Olivera, "Dose calibration of nonconventional treatment systems applied to helical tomotherapy," Med. Phys. 32, 570-577 (2005).

${ }^{12}$ R. E. Drzymala, R. C. Wood, and J. Levy, "Calibration of the Gamma Knife using a new phantom following the AAPM TG51 and TG21 protocols," Med. Phys. 35, 514-521 (2008).

${ }^{13}$ R. Alfonso, P. Andreo, R. Capote, M. S. Huq, W. Kilby, P. Kjall, T. R. Mackie, H. Palmans, K. Rosser, J. Seuntjens, W. Ullrich, and S. Vatnitsky, "A new formalism for reference dosimetry of small and nonstand- 
ard fields," Med. Phys. 35, 5179-5186 (2008).

${ }^{14}$ P. Andreo, D. T. Burns, K. Hohlfeld, M. S. Huq, T. Kanai, F. Laitano, V. G. Smyth, and S. Vynckier, "Absorbed dose determination in external beam radiotherapy," International Atomic Energy Agency (IAEA), Technical Report Series No. 398 (Vienna, 2000).

${ }^{15}$ P. Francescon, S. Cora, and C. Cavedon, "Erratum: 'Total scatter factors of small beams: A multidetector and Monte Carlo study' [Med Phys 35, 504-513 (2008)],” Med. Phys. 37, 947 (2010).

${ }^{16}$ A. J. Scott, A. E. Nahum, and J. D. Fenwick, "Using a Monte Carlo model to predict dosimetric properties of small radiotherapy photon fields," Med. Phys. 35, 4671-4684 (2008).

${ }^{17}$ M. Westermark, J. Arndt, B. Nilsson, and A. Brahme, "Comparative dosimetry in narrow high-energy photon beams," Phys. Med. Biol. 45, 685702 (2000).

${ }^{18} \mathrm{C}$. Antypas and E. Pantelis, "Performance evaluation of a CyberKnife G4 image-guided robotic stereotactic radiosurgery system," Phys. Med. Biol. 53, 4697-4718 (2008).

${ }^{19} \mathrm{O}$. A. Sauer, "Determination of the quality index $(\mathrm{Q})$ for photon beams at arbitrary field sizes," Med. Phys. 36, 4168-4172 (2009).

${ }^{20} \mathrm{BJR}$, "Central axis depth dose data for use in radiotherapy," Br. J. Radiol., Suppl. 25, 62-107 (1996).

${ }^{21}$ P. Sharpe and J. Sephton, "Therapy level alanine dosimetry at the NPL," in Proceedings of the 216th PTB Seminar: Alanine Dosimetry for Clinical Applications, Braunschweig, Germany, 2006

${ }^{22}$ M. Anton, R.-P. Kapsch, M. Krystek, and F. Renner, "Response of the alanine/ESR dosimetry system to MV x-rays relative to ${ }^{60} \mathrm{Co}$ radiation," Phys. Med. Biol. 53, 2753-2770 (2008).

${ }^{23}$ G. G. Zeng, M. R. McEwen, D. W. O. Rogers, and N. V. Klassen, "An experimental and Monte Carlo investigation of the energy dependence of alanine/EPR dosimetry: I Clinical x-ray beams," Phys. Med. Biol. 49, 257-270 (2004).

${ }^{24}$ International Organization for Standardization, "Guide to the expression of uncertainty in measurement (GUM)," 1995.

${ }^{25}$ P. Castro, F. Garcia-Vicente, C. Minguez, A. Floriano, D. Sevilliano, L. Perez, and J. J. Torres, "Study of the uncertainty in the determination of the absorbed dose to water during external beam radiotherapy calibra- tion," J. Appl. Clin. Med. Phys. 9, 70-86 (2007).

${ }^{26}$ A. Moutsatsos, L. Petrokokkinos, P. Karaiskos, P. Papagiannis, E. Georgiou, K. Dardoufas, P. Sandilos, M. Torrens, E. Pantelis, I. Kantemiris, L. Sakelliou, and I. Seimenis, "Gamma knife output factor measurements using VIP polymer gel dosimetry," Med. Phys. 36, 4277-4287 (2009).

${ }^{27}$ S. Devic, J. Seuntjens, E. Sham, E. B. Podgorsak, C. R. Schmidtlein, A. S. Kirov, and C. G. Soares, "Precise radiochromic film dosimetry using a flat-bed document scanner," Med. Phys. 32, 2245-2253 (2005).

${ }^{28}$ L. B. Leybovich, A. Sethi, and N. Dogan, "Comparison of ionization chambers of various volumes for IMRT absolute dose verification," Med. Phys. 30, 119-123 (2003).

${ }^{29}$ E. Pappas, L. Petrokokkinos, A. Angelopoulos, T. G. Maris, M. Kozicki, I. Dalezios, and V. Kouloulias, "Relative output factor measurements of a $5 \mathrm{~mm}$ diameter radiosurgical photon beam using polymer gel dosimetry," Med. Phys. 32, 1513-1520 (2005).

${ }^{30}$ M. Stasi, B. Baiotto, G. Barboni, and G. Scielzo, "The behavior of several microionization chambers in small intensity modulated radiotherapy fields," Med. Phys. 31, 2792-2795 (2004).

${ }^{31}$ X. R. Zhu, J. J. Allen, J. Shi, and W. E. Simon, "Total scatter factors and tissue maximum ratios for small radiosurgery fields: Comparison of diode detectors, a parallel-plate ion chamber, and radiographic film," Med. Phys. 27, 472-477 (2000).

${ }^{32}$ G. Xiong and D. W. O. Rogers, "Relationship between \%dd(10) and stopping-power ratios for flattening filter free accelerators: A Monte Carlo study," Med. Phys. 35, 2104-2109 (2008).

${ }^{33}$ D. M. González-Castaño, G. H. Hartmann, F. Sanchez-Doblado, F. Gomez, R. P. Kapsch, J. Pena, and R. Capote, "The determination of beam quality correction factors: Monte Carlo simulations and measurements," Phys. Med. Biol. 54, 4723-4741 (2009).

${ }^{34}$ S. Agostinelli, S. Garelli, M. Piergentili, and F. Foppiano, "Response to high-energy photons of PTW31014 PinPoint ion chamber with a central aluminum electrode," Med. Phys. 35, 3293-3301 (2008).

${ }^{35}$ F. Crop, N. Reynaert, G. Pittomvils, L. Paelink, C. De Wagter, L. Vakaet, and H. Thierens, "The influence of small fields sizes, penumbra spot size and measurement depth on perturbation factors for microionization chambers," Phys. Med. Biol. 54, 2951-2969 (2009). 